TIFR/TH/94-24

IC/94/147

hep-th 9407015

\title{
BOUNDARY DYNAMICS IN DILATON GRAVITY
}

\author{
Sumit R. Das \\ Tata Institute of Fundamental Research \\ Homi Bhabha Road, Bombay 400005, INDIA \\ e-mail:das@theory.tifr.res.in \\ and \\ Sudipta Mukherji \\ International Centre for Theoretical Physics \\ I-34100 Trieste, ITALY \\ e-mail:mukherji@ictp.trieste.it
}

\begin{abstract}
We study the dynamics of the boundary in two dimensional dilaton gravity coupled to $N$ massless scalars. We rederive the boundary conditions of [1] and [3] in a way which makes the requirement of reparametrization invariance and the role of conformal anomaly explicit. We then study the semiclassical behaviour of the boundary in the $N=24$ theory in the presence of an incoming matter wave with a constant energy flux spread over a finite interval. There is a critical value of the matter energy density below which the boundary is stable and all the matter is reflected back. For energy densities greater than this critical value there is a similar behaviour for small values of the total energy thrown in. However, when the total energy exceeds another critical value the boundary exhibits a runaway behaviour and the spacetime develops singularities and horizons.
\end{abstract}




\section{Introduction}

In a recent paper [1], we have studied a model of two dimensional gravity and massless matter field with a boundary in space time [2, 3]. The reason for introducing such a boundary in the model is many-fold. In the two dimensional reduction of four dimensional spherically symmetric space time such a boundary may be thought as the origin of the radial coordinate. Moreover semiclassical studies of dilaton gravity models in two dimensions inevitably lead to a boundary beyond which the theory becomes strongly coupled. In a ficucial coordinate system $(u, v)$ the boundary may be chosen to be the line $u=v$. We will refer to this coordinate system as the "fixed boundary gauge". In these coordinates the conformal factor of the metric and the dilaton are independent fields. However, $u$ and $v$ are not asymptotically minkowskian. Alternatively we can work in Kruskal coordinates which are simply related to the asymptotically minkowskian coordinates. In these coordinates the dilaton is equal to the liouville mode and the boundary is dynamical. Its world line is determined by the matter energy momentum tensor, through a boundary equation derived in [1, 3]. In the fixed boundary gauge the same equation determines the relationship between the dilaton and the liouville mode.

In the full quantum theory the boundary undergoes quantum fluctuations which are determined by the boundary equation, which has to be now regarded as an operator equation. (In the fixed boundary gauge, these are the dilaton and liouville fields which fluctuate.) The dilaton-gravity sector of the theory has no physical propagating degrees of freedom, but the degree of freedom corresponding to the boundary survives. Thus we get an idea about the strength of quantum gravity in such simple model by studying boundary fluctuations, as done in [困].

In some sense the boundary acts as a model for the high energy, strong coupling physics of the problem. Just as in the Callan-Rubakov effect we would like to model the high energy physics by suitable boundary conditions. The boundary conditions must respect some minimum requirements, like conservation of charges which should be conserved. In general such minimal requirements may not be restrictive enough to enable us to get insight into the real problem. However, it is also common that minimal requirements turn out to be rather restrictive and allow one to derive "model indpendent" consequences. The boundary conditions which arise in the Callan-Rubakov 
effect is such an example [4].

In the present problem, the boundary conditions must respect conservation of energy momentum and the general covariance of the underlying theory. In a gauge fixed theory the latter means that the reparametrization constraints must be obeyed (in a conformal gauge this is the same as maintaining conformal invariance).

The most general boundary conditions which respect these requirements were derived in [3, 1]. The conditions of general covariance and energymomentum conservation turn out to be rather restrictive. Assuming that the matter is reflected off the boundary separately, the boundary conditions in the dilaton gravity sector is characterized by a single parameter $\beta$, which is related to the value of the dilaton field at the boundary. In [1] a "boundary equation" was derived starting from the fixed boundary gauge. The behavior of the boundary and the resulting space-time structure is similar for all nonzero values of $\beta$, while $\beta=0$ (which are the boundary conditions of [6, 2] ) is a rather singular limit. In fact the boundary conditions arise dynamically when one adds a boundary cosmological constant term proportional to $\beta$ to the two dimensional action [7, 8], which immediately explains why $\beta=0$ is rather special. Our model is in fact similar to the boundary conformal field theories considered recently in [5].

In [1] the semiclassical boundary equation was solved in the $N=24$ theory for an incident shock wave. For $\beta \neq 0$ there is a critical value of the strength of the shock wave, i.e. the total energy. Below this critical value the boundary has a stable timelike evolution and no black hole is formed. When the energy exceeds the critical value the boundary runs away with ever increasing acceleration, approaching the speed of light. At the same time a black hole is formed which subsequently evaporates. The stability of the boundary for small disturbances have been recently analyzed in [7] for arbitrary $N$ and an intriguing proposal based on the similarity of the model with open string theory has been put forward for the $N=\infty$ theory in [8].

Note that a limit on the strength of the shock wave does not set any limit on the energy density for an aribitrary pulse. The $\beta=0$ theory, which is essentially the RST model, can be solved for arbitrary distribution of incoming matter energy and a black hole is formed when the energy density exceeds a critical value. However, in this model the boundary becomes spacelike whenever this critical value is exceeded. It is of interest to know the behavior of the $\beta \neq 0$ model for more general incoming matter distribution 
and ask whether there is some critical value for the energy density as well.

In this letter we first rederive the boundary condition and the boundary equation for general $N$ in the Kruskal gauge. This derivation makes the role of reparametrization invariance and the anomaly in the boundary conditions explicit. We then solve the semiclassical boundary equation for $N=24$ for an incoming matter distribution which consists of a constant energy density in a finite interval of retarded time. We find that for the boundary to become unstable and a black hole to form, there is a critical value of the energy density as well as the total energy.

\section{The Boundary Equation}

The classical action of the model is given by

$$
S=\frac{1}{2 \pi} \int d u d v \sqrt{g}\left[e^{-2 \phi}\left(R-4(\nabla \phi)^{2}-4 \lambda^{2}+\frac{1}{2}(\nabla f)^{2}\right]\right.
$$

where $g_{a b}(u, v), \phi(u, v)$ and $f^{i}(u, v)$ are the two dimensional metric, dilaton and the matter fields. $R$ is the curvature scalar and $\lambda$ is the comological constant which we set to one by properly choosing the scale.

We will use the conformal gauge where $g_{a b}=e^{2 \rho} \eta_{a b}$. Since the matter is conformally coupled its quantum effects are entirely contained in the Weyl anomaly which means that there is a liouville term in the action. There are additional liouville terms coming from the measures of integration of the conformal mode $\rho$, the dilaton and the ghosts. These measures are chosen so as to ensure that the Hawking radiation consists of only physical propagating particles, which are the matter fields $f^{i}$. Following RST one can use the freedom to add a local counterterm to make the semiclassical model solvable. The resulting action may be written as

$$
\left.S=\frac{\kappa}{\pi} \int d u d v\left[2 \Omega \partial_{u} \partial_{v} \hat{\rho}-e^{2 \hat{\rho}}-2\left(1-\frac{1}{\kappa}\right) \partial_{u} \hat{\rho} \partial_{v} \hat{\rho}+\frac{1}{2} \partial_{u} f^{i} \partial_{v} f^{i}+\text { (ghosts }\right)\right]
$$

where we have defined the fields

$$
\Omega=\frac{1}{\kappa}\left[e^{-2 \phi}+\kappa \phi\right] \quad \hat{\rho}=\rho-\phi
$$

and $\kappa=\frac{N}{24}$. 
It is also possible to write down models which are exactly conformally invariant [9, 10]. These models have an action which is identical to (2)). However the expressions for $\Omega$ and $\hat{\rho}$ in terms of $\phi$ and $\rho$ are different. The boundary conditions and the boundary equation which we will derive are valid for these theories as well. However the interpretation of the solution in terms of the space-time structure would be different.

The equations of motion following from (2) are very simple

$$
\partial_{u} \partial_{v} \hat{\rho}=\partial_{u} \partial_{v} f^{i}=0 \quad \partial_{u} \partial_{v} \Omega=-e^{2 \hat{\rho}}
$$

Following [2] we introduce two chiral fields $X^{+}(u)$ and $X^{-}(v)$ and solve the equation for $\hat{\rho}$

$$
e^{2(\rho-\phi)}=\partial_{u} X^{+}(u) \partial_{v} X^{-}(v)
$$

The equation for $\Omega$ has the general solution

$$
\Omega=-X^{+}(u) X^{-}(v)+g^{+}(u)+g^{-}(v)+K
$$

where $K$ is a constant and $g^{ \pm}$are arbitrary chiral functions. We can trade these functions for two other chiral fields $Y^{+}(v)$ and $Y^{-}(u)$ which are defined as

$$
\partial_{u} g^{+}(u)=Y^{-}(u) \partial_{u} X^{+}(u), \quad \partial_{v} g^{-}(v)=Y^{+}(v) \partial_{v} X^{-}(v),
$$

In the gauge fixed theory the requirement of general covariance of the underlying model is imposed by setting the total energy momentum tensor to be zero

$$
T_{u u}^{g}+T_{u u}^{m}+T_{u u}^{g h}=0 \quad T_{v v}^{g}+T_{v v}^{m}+T_{v v}^{g h}=0
$$

where $T^{g}, T^{m}, T^{g h}$ stand for the graviton-dilaton, matter and the ghost energy momentum tensors respectively. In what follows, the ghosts completely decouple. The matter part $T^{m}$ is standard, while the graviton-dilaton part has the following expressions

$$
\begin{aligned}
& T_{u u}^{g}=\kappa \partial_{u} Y^{-} \partial_{u} X^{+}+\frac{\hat{\kappa}}{2}\left[\left(\partial_{u} \log \partial_{u} X^{+}\right)^{2}-2 \partial_{u}^{2} \log \partial_{u} X^{+}\right] \\
& T_{v v}^{g}=\kappa \partial_{v} Y^{+} \partial_{v} X^{-}+\frac{\hat{\kappa}}{2}\left[\left(\partial_{v} \log \partial_{v} X^{-}\right)^{2}-2 \partial_{v}^{2} \log \partial_{v} X^{-}\right]
\end{aligned}
$$

where $\hat{\kappa}=\frac{N-24}{24}$. Note that in (8) above, the matter part $T^{m}$ contains a vacuum energy term coming from normal ordering effects. Alternatively in the 
semiclassical theory the vacuum has to be defined in terms of modes which are positive frequency with respect to asymptotically minkowskian coordinates and there is a term which comes from the anomalous transformation of the energy momentum tensor to some other globally valid coordinate system.

We now derive the general boundary condition. The minimal requirements which the theory with these boundary conditions must satisfy are

1. The dilaton field must be a constant along the boundary

2. The matter must be perfectly reflected from the boundary

3. The reparametrization constraints (8) must be obeyed.

The last condition guarantees that the boundary conditions are consistent with the general covariance of the model.

Consider a timelike boundary in the theory specified by the equation

$$
v=f_{B}(u)
$$

or its inverse equation

$$
u=g_{B}(v)
$$

The condition (2) above and the constraint (8) imply that the total energy momentum tensor must be perfectly reflected. This means that the right moving energy momentum tensor must be set to be equal to the left moving energy momentum tensor after performing a coordinate transformation by the function (11). In doing so one must remember that energy momentum tensors transform anomalously with a Schwarzian derivative term.

We will impose Dirichlet boundary conditions on the matter fields

$$
f^{i}\left(g_{B}(v), v\right)=0
$$

We will also impose similar boundary conditions on the ghosts. This means that general covariance requires that the gravity energy momentum tensor must obey

$$
T_{v v}^{g}(v)=\left(\partial_{v} g_{B}(v)\right)^{2} T_{u u}^{g}\left[g_{B}(v)\right]+\hat{\kappa}\left\{g_{B}(v), v\right\}
$$

where $\{.,$.$\} denotes a Schwarzian derivative$

$$
\{f, x\}=\frac{f^{\prime \prime \prime}}{f^{\prime}}-\frac{3}{2}\left(\frac{f^{\prime \prime}}{f^{\prime}}\right)^{2}
$$


In the bulk theory independent reparametrizations of $u$ and $v$ are still symmetries in the conformal gauge. This is no longer true if we choose the boundary to be a fixed line $u=v$ as in the fixed boundary gauge. However, one of the conformal reparametrizations still remain a symmetry and we can use this to choose $X^{+}(u)=u$. This leaves $X^{-}(v)$ as the dynamical degreee of freedom, which is determined by the constraints. This is the gauge used in [2, 1]. In the semiclassical theory, the constraints determine the function $X^{-}(v)$ in terms of the energy momentum tensor of the matter. In the quantum theory the fluctuations of $X^{-}(v)$ are determined in terms of the matter fluctuations via the operator constraints. Since in this gauge $g_{B}(v)=$ 1 the Schwarzian derivative term vanishes. However the nontriviality of the transformation properties of the energy momentum tenaor are contained in the expression for $T_{v v}^{g}$ in (9).

Alternatively one may fix the Kruskal gauge $X^{+}(u)=u$ and $X^{-}(v)=v$. The boundary cannot be fixed to be some predetermined line any more. Rather the boundary curve is now dynamically determined, as we shall see soon. In the following we will use the Kruskal gauge and accordingly replace the indices $u$ and $v$ by + and - respectively.

The condition that the dilaton is constant along the boundary follows from the solution for $\Omega$ in (6) and (7)

$$
\left(Y^{-}\left(X^{+}\right)-X^{-}\right)+\left.\left(\partial_{+} f_{B}\right)\left(Y^{+}\left(X^{-}\right)-X^{+}\right)\right|_{X^{-}=f_{B}\left(X^{+}\right)}=0
$$

Let us introduce a new quantity

$$
h_{B}\left(X^{+}\right)=\sqrt{\partial_{+} f_{B}\left(X^{+}\right)}
$$

The condition (15) may be satisfied by setting

$$
\begin{aligned}
Y^{+}\left(X^{-}\right) & =g_{B}\left(X^{-}\right)+F\left[h_{B}\left(g_{B}\left(X^{-}\right)\right), X^{-}\right] \\
Y^{-}\left(X^{+}\right) & =f_{B}\left(X^{+}\right)-\frac{F\left[h_{B}\left(X^{+}\right), X^{+}\right]}{h_{B}^{2}}
\end{aligned}
$$

where $F\left[h_{B}(x), x\right]$ is some general functional of $h_{B}(x)$ and a function of $x$.

We now use these expressions for $Y^{ \pm}$to evaluate $T_{++}^{g}, T_{--}^{g}$ in (13) and obtain a functional differential equation for $F$. In the Kruskal gauge one simply has $T_{++}^{g}=\partial_{+} Y^{+}$etc. However since the boundary curve is nontrivial, the term in (13) which involves $\hat{\kappa}$ is nonvanishing. One gets the equation

$$
\partial_{+} F+\partial_{+} h_{B} \frac{\delta F}{\delta h_{B}}=\left(\partial_{+} \log h_{B}\right) F+\frac{\hat{\kappa}}{\kappa}\left[\left(\partial_{+} \log h_{B}\right)^{2}-\partial_{+}^{2} \log h_{B}\right]
$$


This has the most general solution

$$
F\left[h_{B}\left(X^{+}\right), X^{+}\right]=\beta h_{B}\left(X^{+}\right)+\frac{\hat{\kappa}}{\kappa} \partial_{+} \log h_{B}
$$

where $\beta$ is an arbitrary parameter. This completes the derivation of the most general boundary condition which satsifies the requirements stated above. The term involving $\hat{\kappa}$ is clearly the result of the anomalous transformation law of the energy momentum tensor.

Finally, using the above expression for $F$ one may derive the equation of motion of the boundary. This is simply the statement that the total $T_{++}$ vanishes and is given by

$$
\kappa h_{B}^{2}+\kappa \beta \partial_{+} h_{B}+\hat{\kappa} \partial_{+}^{2} \log h_{B}+T_{++}^{m}-\frac{\kappa}{2\left(X^{+}\right)^{2}}=0
$$

The last term is the standard vacuum energy which appears in the Kruskal gauge.

So far we have not specified the value of the dilaton field on the boundary. At this stage this may appear as a second parameter specifying the boundary. However, as we shall se below, this is actually determined by the condition that the vacuum solution of the boundary equation corresponds to flat space with a linear dilaton.

The boundary equation (20) determines the shape of the boundary curve in terms of the matter energy momentum tensor. Once this is known, we can use the expressions for $Y^{-}, Y^{+}$to write the solution for $\Omega$ and hence derive the space-time structure. In the following we will concentrate on the behaviour of the boundary itself.

It is useful to rewrite this equation in terms of the quantity

$$
q_{B}\left(X^{+}\right)=X^{+} h_{B}\left(X^{+}\right)
$$

In terms of $q_{B}$ the equation (20) becomes

$$
\begin{aligned}
\frac{\hat{\kappa}}{\kappa}\left(X^{+} \partial_{+}\right)^{2} \log q_{B}+ & \left(\beta q_{B}-\frac{\hat{\kappa}}{\kappa}\right)\left(X^{+} \partial_{+}\right) \log q_{B} \\
& +\left(q_{B}^{2}-\beta q_{B}+\frac{\hat{\kappa}}{\kappa}-\frac{1}{2}\right)=-\frac{\left(X^{+}\right)^{2}}{\kappa} T_{++}^{M}
\end{aligned}
$$

As noted in [7] this represents the equation of motion for a particle driven with some external force and subjected to some damping. In vacuum $T_{++}^{M}=0$ 
and the external force is the gradient of a potential. Consequently one may expect that the ground state is characterized by a constant $g_{B}$ which is the minimum of this potential. However, for this to occur for real $g_{B}$ one must have $\beta^{2}+2>4 \frac{\hat{\kappa}}{\kappa}$. For general $N$ one cannot, therefore, choose $\beta=0$ [3] . Furthermore for the vacuum solution to be stable under small deviations, one must have $\hat{\kappa} \geq 0$ and $\beta>\frac{\sqrt{2} \hat{\kappa}}{\kappa}$.

In the rest of the paper we shall limit ourselves to $N=24$ for which the vacuum is perturbatively stable.

\section{Semiclassical Solution with a finite dura- tion wave}

We now investigate the stability of the boundary for arbitrary incoming energy and energy density for $N=24$. Even in this case, we have not been able to solve the equation (22) for arbitrary $T_{++}$. However to understand the nature of threshold of instability and black hole formation we only need to have a situation where we can tune the energy density and the total energy independently. We will thus solve the above equation for an incoming matter wave which has a constant energy density for a finite interval of retarded time. Thus we have a matter energy momentum tensor of the form

$$
T_{++}^{M}=\frac{\alpha}{2\left(X^{+}\right)^{2}}\left[\theta\left(X^{+}-1+\epsilon\right)-\theta\left(X^{+}-1-\epsilon\right)\right]
$$

which corresponds to a constant energy density $\frac{\alpha}{2}$ in the interval $1-\epsilon<$ $X^{+}<1+\epsilon$. The total energy is thus $M=\alpha \epsilon$.

In the following we will name the various regions of the $X^{+}$space as follows (i) Region I : $X^{+}<1-\epsilon$ (ii) Region II $1-\epsilon<X^{+}<1+\epsilon$ (iii) Region III $X^{+}>1+\epsilon$.

In Regions I and II the solution is given by

$$
q_{B}\left(X^{+}\right)=\frac{1}{2}\left[\beta+\sqrt{\Delta} \frac{\left(X^{+}\right)^{\delta}+d}{\left(X^{+}\right)^{\delta}-d}\right]
$$

where $d$ is an integration constant. In (24) we have made the following definitions

$$
\Delta=\beta^{2}+2 \quad \delta=\frac{\sqrt{\Delta}}{\beta}
$$


We want to have initial conditions such that the space time is in the vacuum, and the boundary has a vacuum hyperbolic solution discussed above, before any matter has come in. This means that in region I we have to choose $d=0$. This determines the value of the dilaton field on the boundary to be the quantity [迆]

$$
\left.e^{-2 \phi}\right|_{\text {boundary }}=\frac{1}{2}[1+\beta(\beta+\sqrt{\Delta})]
$$

Thus the value of the dilaton field is also determined in terms of the parameter $\beta$ as mentioned above.

The nature of the solution in region II is qualitatively different for small and large $\alpha$ regardless of the value of $\epsilon$. Let us define $\alpha_{0}=\frac{\Delta}{2}$.

\section{$3.1 \alpha<\alpha_{0}$}

For $\alpha<\alpha_{0}$ the solution in region II is given by

$$
q_{B}=\frac{1}{2}\left[\beta+\sqrt{\Upsilon} \frac{\left(X^{+}\right)^{\eta}+d_{I I}}{\left(X^{+}\right)^{\eta}-d_{I I}}\right]
$$

where we have defined

$$
\Upsilon=\Delta-2 \alpha \quad \eta=\frac{\sqrt{\Upsilon}}{\beta}
$$

The constant of integration $d_{I I}$ is determined by requiring that $q_{B}$ is continuous across $X^{+}=1-\epsilon$. This gives

$$
d_{I I}=(1-\epsilon)^{\eta} \frac{\sqrt{\Delta}-\sqrt{\Upsilon}}{\sqrt{\Delta}+\sqrt{\Upsilon}}
$$

From the solution (27) it is clear that $q_{B}$ and hence $f_{B}$ will become infinite and the boundary will run away if $\left(X^{+}\right)^{\eta}-d_{I I}=0$ for some value of $X^{+}>(1-\epsilon)$. This requires $d_{I I}>(1-\epsilon)^{\eta}$. However, using the definitions of $\Delta$ and $\Upsilon$ it is clear from (29) that $d_{I I}$ is always less than $(1-\epsilon)^{\eta}$. Thus there cannot be any runaway of the boundary in the region II.

The solution in region III is given by (24) with the integration constant denoted by $d_{I I I}$. This is in turn determined by the solution in region II by matching across $X^{+}=1+\epsilon$, which yields the following equation for $d_{I I I}$

$$
\frac{(1+\epsilon)^{\delta}+d_{I I I}}{(1+\epsilon)^{\delta}-d_{I I I}}=\frac{\sqrt{\Upsilon}}{\sqrt{\Delta}} \frac{(1+\epsilon)^{\eta}+d_{I I}}{(1+\epsilon)^{\eta}-d_{I I}}
$$


Since $d_{I I}<(1-\epsilon)^{\eta}<(1+\epsilon)^{\eta}$ it is clear from (30) that $d_{I I I}<(1+\epsilon)^{\delta}$. The solution (24) then shows that $q_{B}$ cannot blow up anywhere in region III as well.

We thus conclude that for $\alpha<\frac{\Delta}{2}$ the boundary is stable and remains timelike throughout.

\section{$3.2 \alpha>\alpha_{0}$}

For $\alpha>\alpha_{0}$ the solution in Region II is given by

$$
q_{B}=\frac{1}{2}\left[\beta-\sqrt{\Sigma} \tan \left(\mu \log X^{+}+c_{I I}\right)\right]
$$

where we have defined

$$
\Sigma=2 \alpha-\Delta \quad \mu=\frac{\sqrt{\Sigma}}{2 \beta}
$$

The intgration constant $c_{I I}$ is once again determined by matching the solution with the vacuum solution at $X^{+}=1-\epsilon$. This gives

$$
\tan c_{I I}=\frac{\tan \mu \log (1-\epsilon)+\gamma}{\gamma \tan \mu \log (1-\epsilon)-1}
$$

where we have defined

$$
\gamma=\frac{\sqrt{\Delta}}{\sqrt{\Sigma}}
$$

The solution in region III is still given by (24) which we rewrite as

$$
q_{B}\left(X^{+}\right)=\frac{1}{2}\left[\beta+\sqrt{\Delta} \frac{\left(X^{+}\right)^{\delta}+c_{I I I}}{\left(X^{+}\right)^{\delta}-c_{I I I}}\right]
$$

$c_{I I I}$ is determined by matching across $X^{+}=1+\epsilon$ which yields

$$
c_{I I I}=(1+\epsilon)^{\delta} \frac{\left(1+\gamma^{2}\right) \tan \left[\mu \log \left(\frac{1+\epsilon}{1-\epsilon}\right)\right]}{\left(1-\gamma^{2}\right) \tan \left[\mu \log \left(\frac{1+\epsilon}{1-\epsilon}\right)\right]-2 \gamma}
$$

Let us first look for runaways of the boundary in the region III. The solution shows that this can happen if the value of $c_{I I I}$ is such that the denominator in (35) vanishes for a value of $X^{+}>(1+\epsilon)$. This means that 
one must have $c_{I I I}>(1+\epsilon)^{\delta}$. The solution for $c_{I I I}$ then shows that this would require, for $\gamma \geq 1$,

$$
-\frac{2 \gamma}{\gamma^{2}-1}<\tan \left[\mu \log \left(\frac{1+\epsilon}{1-\epsilon}\right)\right]<-\frac{1}{\gamma}
$$

whereas for $\gamma<1$ one must have either

$$
\tan \left[\mu \log \left(\frac{1+\epsilon}{1-\epsilon}\right)\right]>\frac{2 \gamma}{1-\gamma^{2}}
$$

or

$$
\tan \left[\mu \log \left(\frac{1+\epsilon}{1-\epsilon}\right)\right]<-\frac{1}{\gamma}
$$

It may be easily checked that in the limiting case of a shock wave this reproduces the limit on the total energy derived in [1]. This limit corresponds to $\alpha \rightarrow \infty$ and $\epsilon \rightarrow 0$ with the total mass $M=\alpha \epsilon$ kept fixed. Then (35) reduces to the condition $M>\beta \sqrt{\Delta}$.

For finite values of $\alpha$ and $\epsilon$ one has a more complex behaviour, since the various integration constants are periodic functions of $\alpha$ and $\epsilon$. Let us study the behaviour of the boundary for some given value of $\alpha>\alpha_{0}$ and $\beta$ and the extent of the pulse $\epsilon$ increasing from zero. It follows from (37)-(39) that the conditions for runaway in region III are satisfied for $\epsilon$ lying in the range $\epsilon_{n}^{(1)}<\epsilon<\epsilon_{n}^{(2)}$ where

$$
\begin{aligned}
\epsilon_{n}^{(1)} & =\tanh \left\{\frac{1}{2 \mu}\left[\delta_{n} \pi+\tan ^{-1}\left(\frac{2 \gamma}{1-\gamma^{2}}\right)\right]\right\} \\
\epsilon_{n}^{(2)} & =\tanh \left\{\frac{1}{2 \mu}\left[n \pi-\tan ^{-1}\left(\frac{1}{\gamma}\right)\right]\right\}
\end{aligned}
$$

where $n=1,2, \cdots$ and

$$
\delta_{n}=(n-1) \theta(1-\gamma)+n \theta(\gamma-1)
$$

In the above equation the branch of the inverse tangent function has been chosen to be the interval $\left[-\frac{\pi}{2}, \frac{\pi}{2}\right]$. It is easy to check that $\epsilon_{n}^{(1)}<\epsilon_{n}^{(2)}<\epsilon_{n+1}^{(1)}$

The behaviour of the boundary may be now summarized as follows. For $\epsilon<\epsilon_{1}^{(1)}, q_{B}$ is finite and the boundary is stable. For $\epsilon_{1}^{(1)}<\epsilon<\epsilon_{1}^{(2)}$ the boundary runs away in region III. For $\epsilon_{1}^{(2)}<\epsilon<\epsilon_{2}^{(1)}$ the quantity $q_{B}$ diverges 
in region II and there is no divergence in region III. When $\epsilon_{2}^{(1)}<\epsilon<\epsilon_{2}^{(2)}$ a new divergence of $q_{B}$ appears in region III, and the pattern continues.

In the physical problem a divergence of $q_{B}$ results in a runaway of the boundary. Just as in the case of the shock wave analyzed in [1] a singularity develops which is asymptotic to the boundary where it diverges and there is a resulting event horizon. Thus for the physical situation at hand, the critical value of $\epsilon$ for which the boundary starts running away and a black hole is formed is the lowest value of $\epsilon$ for which any of the conditions (37)(38) are satisfied, i.e. $\epsilon_{c r}=\epsilon_{1}^{(1)}$. When $\epsilon$ exceeds a higher value $\epsilon_{1}^{(2)}$ the runaway occurs inside the region of the incident pulse. The other runaways for higher values of $\epsilon$ are irrelevant because they occur in a region which is already behind the singularity and hence not contained in the semiclassical space-time. Profiles of the boundary for fixed value of $\alpha$ and different $\epsilon$ (and hence different mass $M$ ) are shown in the Fig.1 - 3 .

Note that we have kept $\alpha, \beta$ fixed in the above discussion. It may be seen from (40) that $\epsilon_{c r}$ decreases monotonically from 1 at $\alpha=\alpha_{0}$ to zero for infinite

$\alpha$, with $\epsilon_{c r} \rightarrow \frac{\beta \sqrt{\Delta}}{\alpha}$ as $\alpha \rightarrow \infty$. Thus a limit of $\epsilon$ for some given $\alpha$ means that there is a lower limit on the total mass of matter $M_{c r}(\alpha)=\alpha \epsilon_{c r}$ which has been thrown in. For $\alpha=\alpha_{0}$ one has $M_{c r}=\alpha_{0}$. As $\alpha$ increases, $M_{c r}(\alpha)$ first rises to a maximum value and then decreases, eventually becoming asympotic to the constant shock wave value $\beta \sqrt{\Delta}$ for large $\alpha$.

We thus conclude that for the boundary to run away and a black hole to form there is a minimum value of the incoming energy density as well as a minimum value of the total energy.

\section{Outlook}

The fact that the runaway of the boundary occurs at the same threshold at which a black hole singularity is formed in the semiclassical model is significant. While we have demonstrated this in the $N=24$ model, we expect that a similar behaviour would hold for $N \neq 24$ as well. However the differential euqtion in the latter case is higher order and the stability analysis would be significantly different. It would be interesting to get an idea of this by using numerical integration techniques. Some work in this direction has been done in [7].

For the shock wave solution we found in [1] that the runaway of the 
boundary in the semiclassical theory also signals the threshold beyond which an aypmtotic observer measures large quantum fluctuations of the boundary near the horizon. It would be interesting to understand the nature and implications of the boundary flcutuations in a more general setting. We leave that for future investigation.

Acknowledgements: We would like to thank H. Verlinde for discussions. One of us (S.M.) would like to thank Professor Abdus Salam, the International Atomic Energy and UNESCO for hospitality at the International Centre for Theoretical Physics, Trieste. S.R.D. would like to thank the Theoretical Physics Groups of Brown University, Princeton University, Enrico Fermi Institute and Washington University for hospitality during the final stages of this work.

\section{References}

[1] S.R. Das and S. Mukherji, hep-th/9401102, to appear in Physical Review , D50.

[2] E. Verlinde and H. Verlinde, Nuclear Physics, B406 (1993) 43; K. Schoutens, E. Verlinde and H. Verlinde, Physical Review D48 (1993) 2690 .

[3] T. Chung and H. Verlinde, Nuclear Physics, B418 (1994) 305.

[4] C.G. Callan and S.R. Das, Physical Review Letters , 51 (1983) 1155.

[5] C.G. Callan and I. Klebanov, hep-th/9311092; I. Affleck and J. Sagi, hep-th/9311056; C.G. Callan, I. Klebanov, A.W.W. Ludwig and J.M. Maldacena, hep-th/9402113; J. Polchinski and L. Thorlacius, hepth/9404008.

[6] J.G. Russo, L. Susskind and L. Thorlacius, Physical Review , D46 (1992) 3444; Physical Review , D47 (1993) 533.

[7] A. Strominger and L. Thorlacius, hep-th/9405084.

[8] A. Strominger, hep-th/9405094. 
[9] A. Bilal and C.G. Callan, Nuclear Physics, B394 (1993) 73.

[10] S. de Alwis, Physics Letters , B289 278; Physics Letters , B300 (1993) 330; Physical Review D46 5429. 


\section{FIGURES}

Fig.1: Typical boundary curve for $\alpha<\alpha_{0}$ for any $\epsilon$. Even for $\alpha>\alpha_{0}$ and $M<M_{c r}(\alpha)$ the boundary curve is similar. No singularities are formed.

Fig. 2: Boundary curve for $\alpha>\alpha_{0}$ and $M>M_{c r}(\alpha)$. Boundary runs away after the matter infall ends. Cosequently space-time singularity is formed.

Fig. 3: For yet higher value of $M$, i.e. for the corresponding $\epsilon>\epsilon_{c r}^{(2)}$, the boundary runaway occurs in the region of incident pulse. 
This figure "fig1-1.png" is available in "png" format from: http://arxiv.org/ps/hep-th/9407015v2 


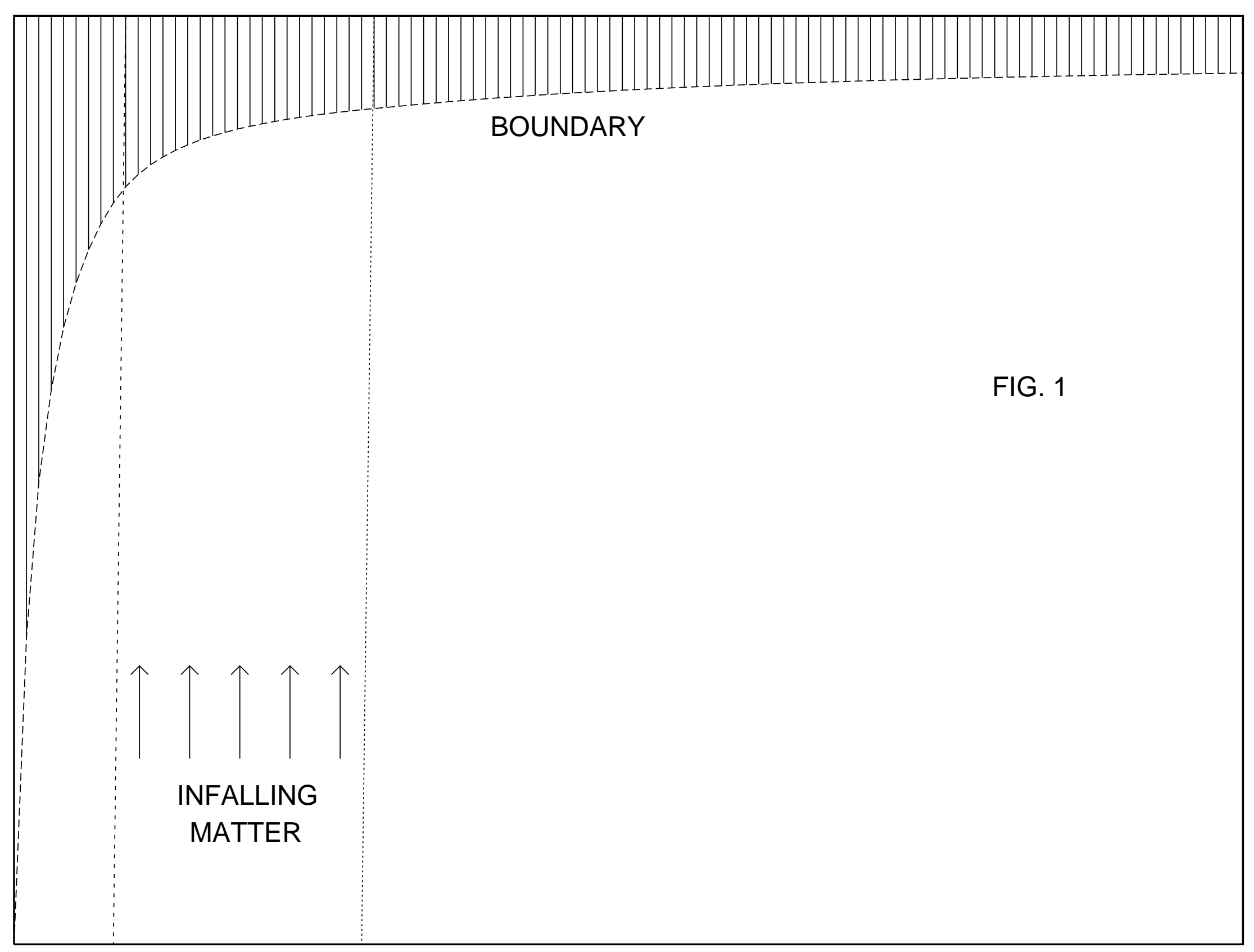


This figure "fig1-2.png" is available in "png" format from: http://arxiv.org/ps/hep-th/9407015v2 
This figure "fig1-3.png" is available in "png" format from: http://arxiv.org/ps/hep-th/9407015v2 


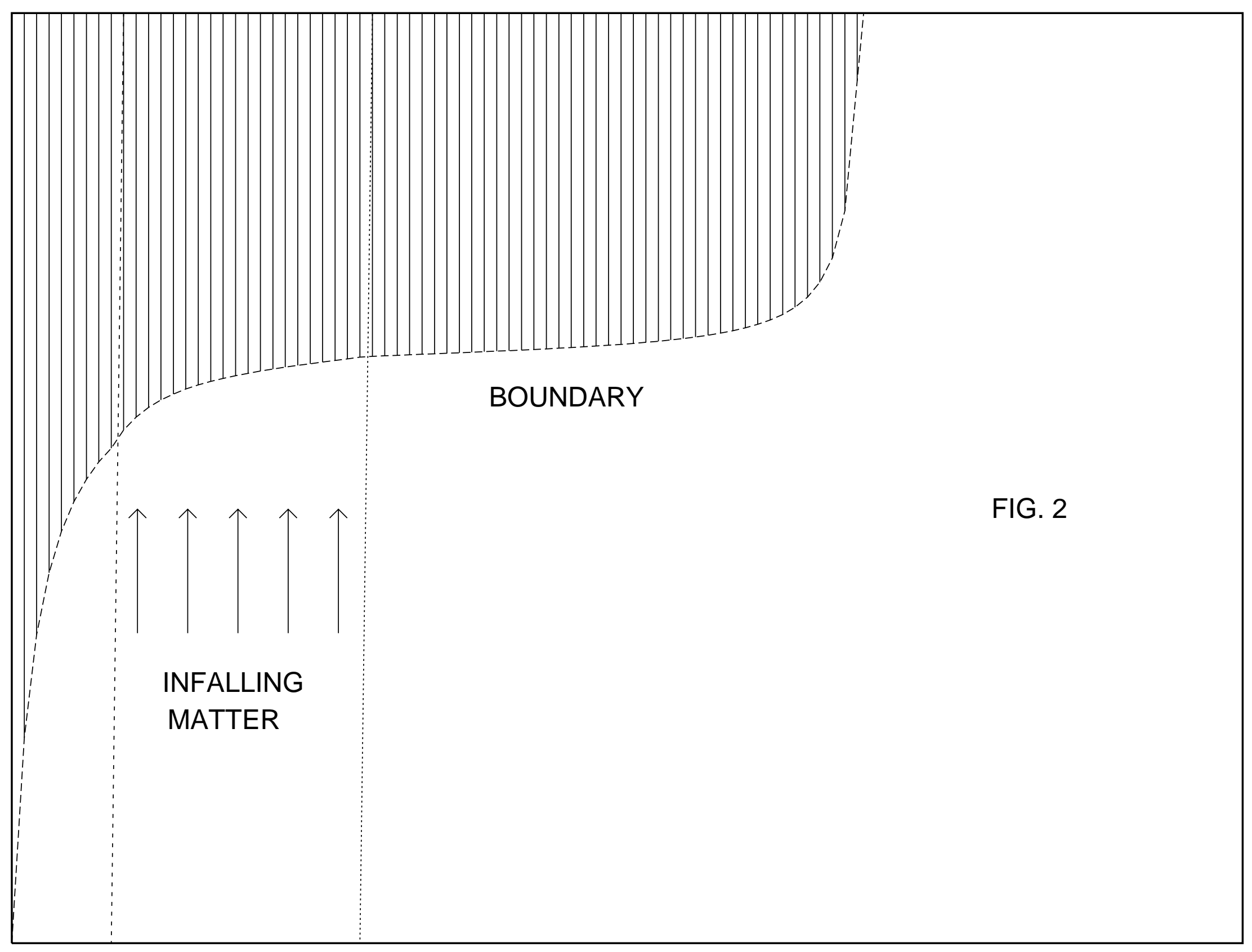




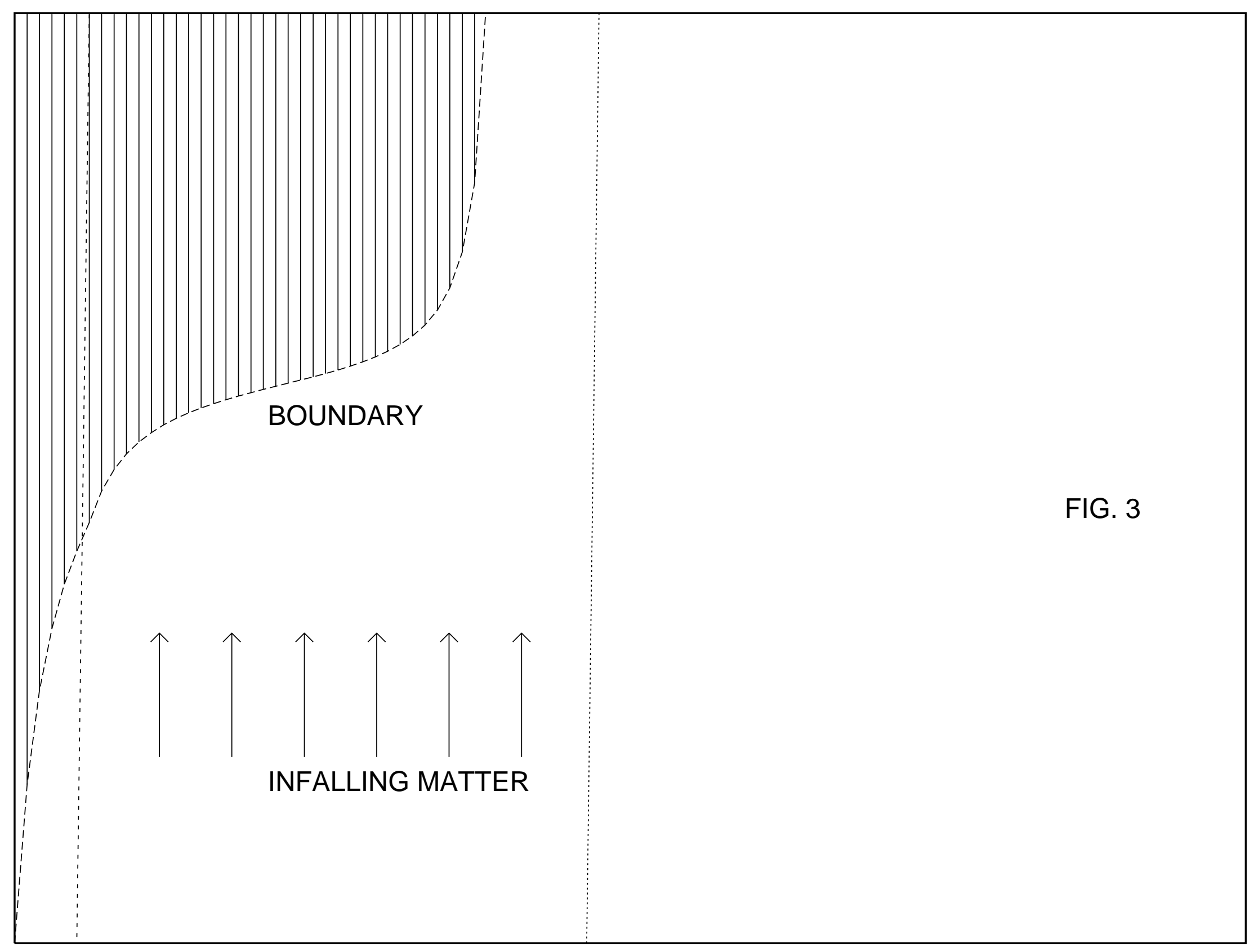

